\title{
Double resonance features in the Raman spectrum of carbon nanotubes
}

\author{
M. L. Sanjuán, ${ }^{1}$ A. Ansón, ${ }^{2}$ and M. T. Martínez ${ }^{2}$ \\ ${ }^{1}$ Instituto de Ciencia de Materiales de Aragón (Universidad de Zaragoza-CSIC), Facultad de Ciencias, Universidad de Zaragoza, 50009 \\ Zaragoza, Spain \\ ${ }^{2}$ Instituto de Carboquímica (CSIC), Miguel Luesma Castán 4, 50018-Zaragoza, Spain
}

(Received 18 March 2004; revised manuscript received 2 September 2004; published 16 November 2004)

\begin{abstract}
A weak feature is observed in the Raman spectra of single-wall carbon nanotube bundles at a frequency between 1410 and $1430 \mathrm{~cm}^{-1}$. The band shifts slightly toward lower frequencies as the excitation energy increases from 2.17 to $2.71 \mathrm{eV}$. An inverse correlation is found between the frequency of the band and that of the average radial-breathing mode, indicating a dependence on the nanotube diameter. The frequency can be extrapolated quadratically to $1487 \mathrm{~cm}^{-1}$ for infinite diameter. Two different hypotheses are considered, ascribing the band to modes around either $K$ or $M$ points of the graphite dispersion relations. The appearance of a band close to $1480 \mathrm{~cm}^{-1}$ in defective graphite and its dependence with the laser energy, as well as the strong sensitivity of high-frequency $K$-point modes to structural or impurity defects, support the interpretation of the mode as arising from for the upper phonon branch of graphite around the $K$ point activated by the doubleresonance mechanism.
\end{abstract}

DOI: 10.1103/PhysRevB.70.201404

PACS number(s): 78.30.Na, 63.22. + m, 81.07.De

In spite of intense theoretical and experimental efforts, lattice dispersion of graphite and other $s p^{2}$ carbons are still controversial, especially as concerns the high-energy branches in the vicinity of the $K$ points. According to different calculations, ${ }^{1-7}$ the $\mathrm{LO}$ and the TO branches cross or do not cross along the $\Gamma-K, K-M$, and $\Gamma-M$ directions and thus the energy of the TO phonon at the $K$ point varies from less than $1300 \mathrm{~cm}^{-1}$ (Refs. 2 and 3) to $1400-1480 \mathrm{~cm}^{-1} .1,8$ Similarly, the LO branch goes along the $\Gamma-K$ direction down to a frequency of $1250 \mathrm{~cm}^{-1}$ (Ref. 1), $1200 \mathrm{~cm}^{-1}$ (Ref. 2), or nearly $1500 \mathrm{~cm}^{-1}$, if the LO band does not cross the TO band. $^{3}$

According to a recent review on lattice dynamics calculations of graphite, ${ }^{5}$ the question of crossing or noncrossing seems to be mainly an issue of the method of calculation. In general, $a b$ initio methods predict a crossing of the upper bands both along the $\Gamma-M$ and $\Gamma-K$ branches, while in force-constant approaches branches along $\Gamma-M$ (and also along $\Gamma-K$, in Ref. 3 ) do not cross. ${ }^{5}$ As regards experimental results, they are not very coincident: In the recent work of Maultzsch et $a .^{2}$ in single crystalline graphite the highfrequency $K$ mode was found at about $1265 \mathrm{~cm}^{-1}$, a frequency much lower than the $1450-1480 \mathrm{~cm}^{-1}$ range found in Raman spectra of certain forms of graphite ${ }^{9}$ and attributed to $i$-TO modes ( $i$ for in plane). ${ }^{1,10}$ Other techniques, such as high-resolution electron energy loss (HREELS) of surface phonons in graphite yield an intermediate value close to $1400 \mathrm{~cm}^{-1} .{ }^{11}$ More reliable data in this frequency region are clearly needed.

In this work we present what we propose is an observation in single-wall nanotubes (SWNT) of the higher-lying band around the $K$ point, activated through the doubleresonance (DR) mechanism, with a frequency between 1410 and $1430 \mathrm{~cm}^{-1}$. Another possible interpretation of the band as arising from the high density of vibrational states around the $M$ point is discussed.

In short, DR Raman scattering is a fourth-order process in which two of the processes involved are resonant with real electronic states. ${ }^{1,3,12-14}$ For first-order phonons, one of the processes is mediated by impurities or defects. Through this mechanism, out-of- $\Gamma$ vibrations may become visible with intensity higher than usual for just disorder-activated modes. In SWNT, forbidden features are expected, besides the allowed and well-known tangential modes at $\nu$ $\approx 1500-1600 \mathrm{~cm}^{-1}$ ( $G$ band), and radial-breathing modes (RBM), at $\nu \approx 100-400 \mathrm{~cm}^{-1}$. DR, for instance, is at the origin of the $D$ band at $\nu \approx 1350 \mathrm{~cm}^{-1},{ }^{12-16}$ and enhances the intensity of second-order bands, such as those labeled as $M^{-}$ and $M^{+}$that appear in the range $1700-1800 \mathrm{~cm}^{-1}$ (Ref. 17) and those close to 2700 or $3200 \mathrm{~cm}^{-1}$, or even higher energies. ${ }^{15,18}$

One of the most characteristic manifestations of DR is the variation of the vibrational frequency with the laser energy, $E_{\mathrm{L}}$, and in fact all the features mentioned above show such a dependence. The activation through DR mechanism of $\mathbf{q}$ $\neq 0$ modes provides a good means of studying the dispersion relations of $s p^{2}$ carbons. In general, a good correspondence is found between the frequency of extra features in carbon nanotubes (CNT) and those of phonon branches in the graphite, the differences being attributed to curvature effects. ${ }^{6,19}$ Conversely, Raman frequencies of modes activated through the DR mechanism have been used to fit graphite dispersion relations in force-constant calculations. ${ }^{3}$

SWNTs were prepared by the arc-discharge method using $\mathrm{Ni} / \mathrm{Y}(4 / 1$ or $2 / 0.5$ at. $\%)$ as catalysts in the graphite precursor. ${ }^{20}$ They form as bundles associated with other carbon phases and metal nanoparticles. Besides the raw SWNT material, SWNTs oxidized in air at temperatures between 300 and $400^{\circ} \mathrm{C}$ for $1 \mathrm{~h}$ have also been used in this work. No structural differences were found between raw and modified materials. The NT diameter range in our samples is $1.30-1.65 \mathrm{~nm}$. Raman experiments were performed with a Dilor XY spectrometer. A microscope with a $\times 50$ objective lens was used; the area explored by the incoming beam being of about $4 \mu^{2}$. Lines of an $\mathrm{Ar}^{+}$laser between 2.41 and $2.71 \mathrm{eV}$, and a dye laser at $2.11-2.17 \mathrm{eV}$, were used as ex- 


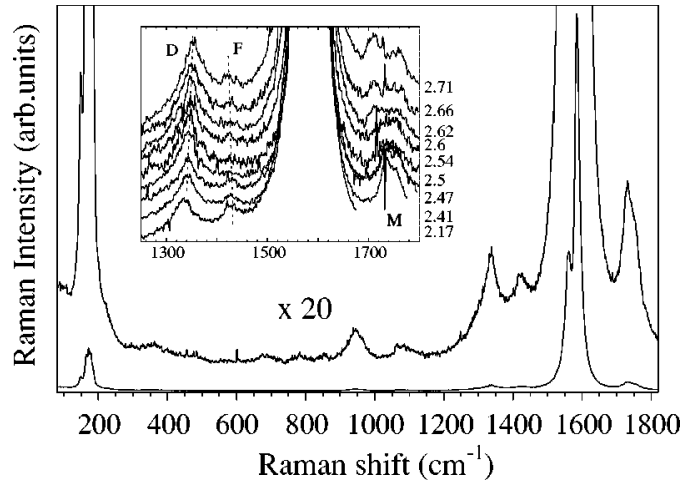

FIG. 1. Raman spectra of SWNT bundles. The long spectrum corresponds to the raw soot material prior to purification and is measured at $E_{\mathrm{L}}=2.41 \mathrm{eV}$. The inset shows the evolution of the $G$-band region with $E_{\mathrm{L}}$ from 2.17 to $2.71 \mathrm{eV}$, as indicated on the right-hand side. The spectra have been normalized to the same $G$-band intensity. The sample used for the spectra of the inset had been heated at $300^{\circ} \mathrm{C}$ for $1 \mathrm{~h}$.

citation light. Power at the sample was always less than $2 \mathrm{~mW}$.

Figure 1 shows a Raman spectrum at $2.41 \mathrm{eV}$ of SWNT bundles. Besides other well-known bands, a weak feature (we shall call it $F$ ) is observed at a frequency $\nu_{\mathrm{F}}$ between 1410 and $1430 \mathrm{~cm}^{-1}$. By comparing many different spectra, a correlation was found between the appearance of the $F$ feature and the $D / G$ relative intensity: the $F$ band is seen when the $\mathrm{D} / \mathrm{G}$ ratio is very low, denoting a high degree of NT crystallinity. In other cases, this feature is obscured by the usually more intense $D$ band. On the other hand, the band is not observed in high quality, highly oriented pyrolytic graphite (HOPG), suggesting that, as for the $D$ band, some defectiveness is necessary to activate it.

The band can be observed, at frequencies close to those reported here and always with very weak intensity, in spectra reported in the literature taken on SWNT samples grown in a wide variety of conditions: by laser ablation with $\mathrm{Ni} / \mathrm{Y}$ catalysts; ${ }^{21}$ by chemical vapor deposition (CVD) with an Fe catalyst; ${ }^{17,22}$ by arc-discharge using as a catalyst $\mathrm{Co},{ }^{23}$ $\mathrm{Ni}-\mathrm{Co},{ }^{24} \mathrm{Ni}-\mathrm{Fe}-\mathrm{Co},{ }^{24}$ or $\mathrm{Ni} / \mathrm{Y}^{25}$ in purified samples with $\mathrm{Ni} / \mathrm{Y}$ catalysts, ${ }^{26}$ etc. It is also found in multiwall nanotubes (MWNT) grown by arc discharge. ${ }^{27}$

As regards other carbonaceous materials, low intensity features have been detected between 1450 and $1500 \mathrm{~cm}^{-1}$ in the Raman spectra of some defective graphites and HOPG. ${ }^{9,18,28,29}$ In Ref. 9 the authors were unable to interpret this feature as any combination of modes. The remarkable dependence of the frequency with elaser $E_{\mathrm{L}}(\Delta \nu$ $\left.=-83 \mathrm{~cm}^{-1} / \mathrm{eV}\right)$ remained also unexplained. The band was attributed in Refs. 1 and 10 to high-frequency $i$-TO modes activated by DR.

In fullerene $C_{60}$ the pentagonal "pinch" mode is seen between 1458 and $1470 \mathrm{~cm}^{-1} \cdot 30,31$ This frequency is quite higher than $\nu_{\mathrm{F}}$ and, moreover, fullerenes would not explain the unambiguous dependence of $\nu_{\mathrm{F}}$ on NT diameter shown below. The possibility that $F$ is a Raman allowed, weakly active mode is also excluded, since the intensity predicted for such modes by a bond polarizability model is
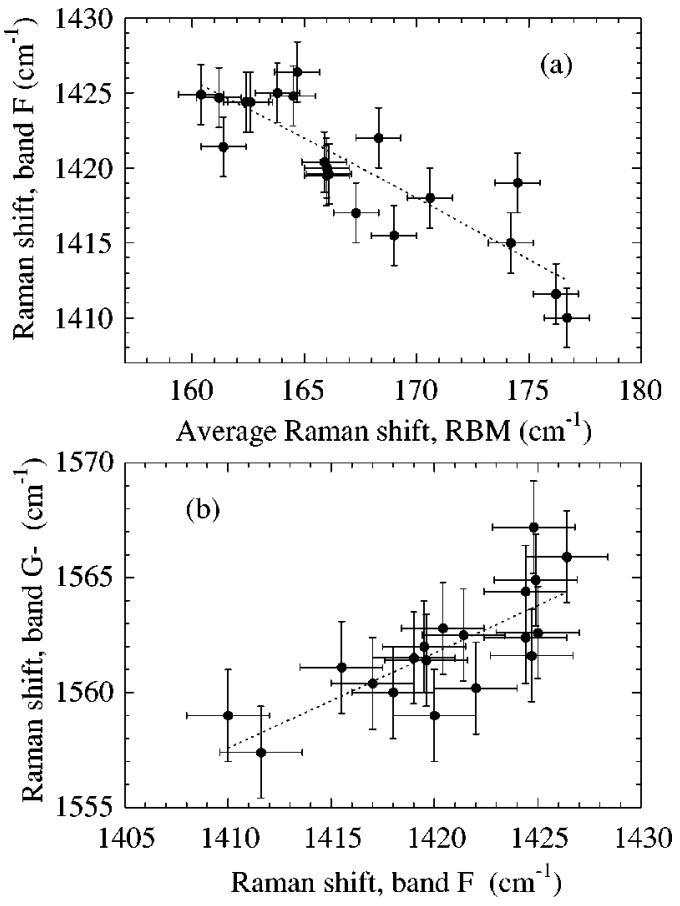

FIG. 2. (a) The frequency of feature $F, \nu_{\mathrm{F}}$, is plotted against the average frequency of the radial-breathing mode, $\nu_{\mathrm{RBM}}$, indicating that a correlation exists between $\nu_{\mathrm{F}}$ and the NT diameter. The dashed line is the fitting of the data to a quadratic expression of the form $\nu_{0}+C^{\prime} \nu_{\mathrm{RBM}}^{x}$ with $\nu_{0}=1487.4 \mathrm{~cm}^{-1}, C^{\prime}=-0.0029331 \mathrm{~cm}^{0.96}$, and $x=1.96$. (b) Correlation between $\nu_{\mathrm{F}}$ and the frequency of the lowest component of the $G$ band, $G^{-}$. The dashed line is only a guide for the eye. Data were taken at $2.41 \mathrm{eV}$.

negligible. ${ }^{32}$ All these observations support the intrinsic origin of the $F$ band as arising from NT.

The inset in Fig. 1 shows the $1200-1800 \mathrm{~cm}^{-1}$ region as a function of laser energy from 2.17 to $2.71 \mathrm{eV}$. The spectra have been normalized to the same $G$-band intensity. The shift of $D$ and $M^{-}, M^{+}$bands upon changing the excitation energy is explained by the DR mechanism. ${ }^{12,15,17}$ The $F$ band, on the contrary, shifts down very slightly with increasing excitation energy $\left(\Delta \nu \approx-10 \mathrm{~cm}^{-1}\right.$ from 2.17 to $\left.2.71 \mathrm{eV}\right)$.

As shown in Fig. 2(a), an inverse correlation exists between $\nu_{\mathrm{F}}$ and the average RBM frequency, $\nu_{\mathrm{RBM}}$ : the higher $\nu_{\mathrm{RBM}}$ (the narrower the tube), the lower $\nu_{\mathrm{F}}$. This trend is similar to that of some high-frequency bands, such as the $D$ band (Ref. 33) or the circumferential component of the $G$ band, $G^{-} .22,34$ Figure 2(b) shows the correlation between $\nu_{\mathrm{F}}$ and $\nu_{\mathrm{G}^{-}}$.

The frequency of diameter-dependent excitations is usually fitted to a law of type $\nu=\nu_{0}+C / d^{x}$, where, for highfrequency bands, $\nu_{0}$ is interpreted as the graphite value since it would be obtained for infinite diameter. ${ }^{33}$ The dashed line in Fig. 2(a) gives the fit to such a dependence or, more precisely, to $\nu_{0}+C^{\prime} \nu_{\mathrm{RBM}}^{x}$, where we have introduced the relation $\nu_{\mathrm{RBM}} \propto 1 / d$. Fitting parameters are $\nu_{0}=1487.4 \mathrm{~cm}^{-1}$, $C^{\prime}=-0.0029331 \mathrm{~cm}^{0.96}$, and $x=1.96$.

Two aspects can be pointed up from the latter results. First, the exponent obtained from the fitting (1.96) is very close to that of $G^{-}$, for which a value of 2 has been given in 
Ref. 22 (although 1.4 in a subsequent work, Ref. 34) suggesting that both features involve closely related vibrations. We note that, according to the calculations of Ref. 6, circumferential vibrations, among the tangential bands, are expected to be the most sensitive to NT diameter. The other remarkable fact is the close similarity between $\nu_{0}\left(1487 \mathrm{~cm}^{-1}\right)$ and the value found in some Raman experiments on graphite $\left(\approx 1480 \mathrm{~cm}^{-1}\right.$ for $\left.E_{\mathrm{L}}=2.41 \mathrm{eV}\right)$.

Two approaches are usually followed to attribute vibrational features of CNT. One is the $a b$ initio calculation of the phonon frequencies and density of states. The other approach, the folding method, assumes that, to a first approximation, NT vibrations will not be very different from those of a graphene sheet or from graphite. Then, the graphite dispersion relations are folded along appropriate directions to account for the one-dimensional (1D) character of CNT. This procedure has been widely used to explain the frequencies and symmetries of allowed modes, mainly in the highfrequency region. In the case of forbidden features a first step is to look at the graphite dispersion relations for modes having similar frequencies, and then propose a mechanism for the activation of such mode. Up to now, all forbidden features (and some allowed ones) of the Raman spectra of CNT are seen to involve the DR mechanism.

Following this procedure, we look for graphite modes close to $1420 \mathrm{~cm}^{-1}$, either in lattice-dynamics calculations or within experimental data. According to different calculations, modes close to $1420 \mathrm{~cm}^{-1}$ can be found either around the $K$ or $M$ points (see Ref. 5 and references therein). As mentioned above, experimental data on high-frequency $K$-point modes show great dispersion, due to the use of different techniques or, more likely, to the high sensitivity of those modes to structural defects, impurities, or even the carboncarbon distance, as suggested in Ref. 5. The observation of a Raman band around $1480 \mathrm{~cm}^{-1}$ in some defective graphites, attributed to $i$-TO modes close to the $K$ point, ${ }^{1,10}$ and its dependendence with $E_{\mathrm{L}}$, clearly indicative of DR being involved, lead in a natural way to consider these modes to be the origin of the $F$ band in CNT, and DR as the mechanism activating it. Moreover, the diameter dependence of $\nu_{\mathrm{F}}$ (Fig. 2 ) is such that it tends, for infinite diameter, toward a value very close to $1480 \mathrm{~cm}^{-1}$. We then consider first this possibility and, later on, an alternative one based on modes around the $M$ point.

Two strong indications of DR being involved in the activation of a given phonon are the frequency shift of the band upon changing the laser energy $\left(\Delta \nu / \Delta E_{\mathrm{L}}\right)$ and a difference between the Stokes and anti-Stokes (AS) positions. Unfortunately, in spite of long accumulation times, the $F$ band could not be detected in the AS spectrum, but there is experimental evidence of such a behavior in the Raman spectrum of MWNT reported in Ref. 27: While the $F$ band is found at $1430 \mathrm{~cm}^{-1}$ in the Stokes spectrum at $E_{\mathrm{L}}=1.58 \mathrm{eV}$, its AS frequency is $1412 \mathrm{~cm}^{-1}$ at the same excitation energy, implying a negative dispersion, as in graphite. ${ }^{9}$

The dispersion of the $F$ band is small and negative, $\Delta \nu / \Delta E_{\mathrm{L}}=-18.5 \mathrm{~cm}^{-1} / \mathrm{eV}$. This value is much smaller than that of the $1480-\mathrm{cm}^{-1}$ band in PG $\left(-83 \mathrm{~cm}^{-1} / \mathrm{eV}\right),{ }^{9}$ but close to the value predicted for the $i$-TO band in Ref. 1 $\left(-11 \mathrm{~cm}^{-1} / \mathrm{eV}\right)$. However, the dispersion of any diameter- dependent band in NT bundles must be taken with precaution, since it involves two different contributions: One is due to the selective resonant excitation of different NT as $E_{\mathrm{L}}$ runs along the Kataura plot; ${ }^{35}$ the other is due to the DR mechanism, according to which the phonons matching the resonance condition vary as $E_{\mathrm{L}}$ is varied. The first contribution is absent in graphite or isolated NT, but can be important in NT bundles if the diameter distribution is wide. As far as one remains within a given $E_{\mathrm{nn}}$ band of van Hove singularities, lower diameters are resonantly excited when $E_{\mathrm{L}}$ is increased. Since $\nu_{\mathrm{F}}$ decreases for decreasing diameter (see Fig. 2), lower $\nu_{\mathrm{F}}$, on the average, would be expected for increasing $E_{\mathrm{L}}$, as observed. However, the situation is not so clear when the NT content is such that several $E_{\text {nn }}$ transitions can be excited. From the diameter range present in our samples $(1.30-1.65 \mathrm{~nm})$, and for $E_{\mathrm{L}}$ between 2.1 and $2.7 \mathrm{eV}$, it derives that $E_{33}^{\mathrm{s}}$ and $E_{44}^{\mathrm{s}}$ transitions may be excited, ${ }^{35}$ but the average diameter being excited, $d_{\mathrm{m}}$, does not show a monotonic behavior: From 2 to $2.2 \mathrm{eV}$ only $E_{33}^{\mathrm{s}}$ transitions are excited, and $d_{\mathrm{m}}$ decreases. As $E_{\mathrm{L}}$ approaches $2.4 \mathrm{eV}$, the $E_{44}^{\mathrm{s}}$ transitions of wider NTs are progresivelly excited and $d_{\mathrm{m}}$ shifts upwards. This holds up to about $2.6 \mathrm{eV}$; beyond this energy narrower tubes are excited in both $E_{33}^{\mathrm{s}}$ and $E_{44}^{\mathrm{s}}$ bands, which yields again a down shift of $d_{\mathrm{m}}$. This nonmonotonic behavior of $d_{\mathrm{m}}$ is observed in the RBM spectrum, but not for the $F$ band, suggesting that these effects are averaged out due to the peculiarities of the DR mechanism: two resonant processes instead of only one, chirality dependence of the excited vibrational frequency. Another important detail is that the outgoing resonance condition is different for RBM and for $F$ modes, as their frequencies differ by $0.16 \mathrm{eV}$, so that, in fact, different NT are resonantly excited when measuring each of these regions. From our data we can only say that, as regards the dispersion effect, our results are compatible with the prediction of DR, though they do not constitute, by its own, a proof of that mechanism. Only measurements in isolated SWNT will be able to separate the spring and DR contributions to the $F$-band dispersion.

We now consider another possible interpretation of feature $F$ as coming from the TO branches around the $M$ point, whose flatness results in a very high density of states at frequencies about $1400 \mathrm{~cm}^{-1}$. The $F$ band might come from this high density of states, without DR being involved at all. However, noncenter modes are not Raman active unless there exists a mechanism that brings them to zone center, either through zone folding or through periodicity breaking. Moreover, even if at $\Gamma$ point, a high density of states does not guarantee a high Raman activity. In the case of CNT, the $M$ point is not systematically folded to $\Gamma$ and, even when it is, it does not provide Raman-active modes, so that another mechanism must be proposed to activate those modes. $M$-point phonons cannot participate in DR processes for laser energies between 2 and $3 \mathrm{eV}$, since electronic gaps at the $M$ point exceed $5 \mathrm{eV}$, so that DR is excluded. One possible mechanism would be the presence of defects in the NTs, but without the concurrence of DR, and due to the low laser power used in the experiments, its activity would be probably very low. We note again that the $F$ band is not observed in nondefective HOPG, thus confirming that some defectivity is required. If the band came from modes around the $M$ 
point, no dispersion with $E_{\mathrm{L}}$ would be expected, neither for graphite nor for NTs.

In summary, considering the ensemble of experimental results presented here for SWNT bundles and those reported in other works for graphite and MWNT, we attribute feature $F$ as coming from high-frequency bands of the graphite dispersion relations around the $K$ point, activated by the DR mechanism. If this attribution is correct, it provides one more confirmation of the idea that all forbidden bands observed in Raman spectra of CNT are activated through DR. Resonance experiments in isolated NTs are clearly needed to verify the dispersion behavior of the band. As regards phonon energies we conclude that fitting graphite dispersion relations to experimental data must be done with great care, since the dispersion of values found for different materials and techniques may yield somewhat erroneous conclusions.

We acknowledge financial support from project MAT2001-3713-C04. A. A. thanks the CSIC Fuel Cell Network for financial support.
${ }^{1}$ R. Saito, A. Jorio, A. G. Souza Filho, G. Dresselhaus, M. S. Dresselhaus, and M. A. Pimenta, Phys. Rev. Lett. 88, 027401 (2002).

${ }^{2}$ J. Maultzsch, S. Reich, C. Thomsen, H. Requardt, and P. Ordejón, Phys. Rev. Lett. 92, 075501 (2004).

${ }^{3}$ A. Grüneis, R. Saito, T. Kimura, L. G. Cançado, M. A. Pimenta, A. Jorio, A. G. Souza Filho, G. Dresselhaus, and M. S. Dresselhaus, Phys. Rev. B 65, 155405 (2002).

${ }^{4}$ R. Al-Jishi and G. Dresselhaus, Phys. Rev. B 26, 4514 (1982).

${ }^{5}$ L. Wirtz and A. Rubio, Solid State Commun. 131, 141 (2004).

${ }^{6}$ O. Dubay and G. Kresse, Phys. Rev. B 67, 035401 (2003).

${ }^{7}$ R. Nicklow, N. Wakabayashi, and H. G. Smith, Phys. Rev. B 5, 4951 (1972).

${ }^{8}$ P. Lespade, R. Al-Jishi, and M. S. Dresselhaus, Carbon 20, 427 (1982).

${ }^{9}$ Y. Kawashima and G. Katagiri, Phys. Rev. B 52, 10053 (1995).

${ }^{10}$ R. Saito, A. Jorio, A. G. Souza Filho, A. Grueneis, M. A. Pimenta, G. Dresselhaus, and M. S. Dresselhaus, Physica B 323, 100 (2002).

${ }^{11}$ S. Siebentritt, R. Pues, K.-H. Rieder, and A. H. Shikin. Phys. Rev. B 55, 7927 (1997).

${ }^{12}$ C. Thomsen and S. Reich, Phys. Rev. Lett. 85, 5214 (2000).

${ }^{13}$ J. Kürti, V. Zólyomi, A. Grüneis, and H. Kuzmany, Phys. Rev. B 65, 165433 (2002).

${ }^{14}$ Ge. G. Samsonidze, R. Saito, A. Jorio, A. G. Souza Filho, A. Grüneis, M. A. Pimenta, G. Dresselhaus, and M. S. Dresselhaus, Phys. Rev. Lett. 90, 027403 (2003).

${ }^{15}$ L. G. Cançado, M. A. Pimenta, R. Saito, A. Jorio, L. O. Ladeira, A. Grueneis, A. G. Souza-Filho, G. Dresselhaus, and M. S. Dresselhaus, Phys. Rev. B 66, 035415 (2002).

${ }^{16}$ J. Maultzsch, S. Reich, and C. Thomsen, Phys. Rev. B 64, 121407(R) (2001).

${ }^{17}$ V. W. Brar, Ge. G. Samsonidze, M. S. Dresselhaus, G. Dresselhaus, R. Saito, A. K. Swan, M. S. Ünlü, B. B. Goldberg, A. G. Souza Filho, and A. Jorio, Phys. Rev. B 66, 155418 (2002).

${ }^{18}$ P. Tan, Y. Deng, and Q. Zhao, Phys. Rev. B 58, 5435 (1998).

${ }^{19}$ D. Sánchez-Portal, E. Artacho, J. M. Soler, A. Rubio, and P. Ordejón, Phys. Rev. B 59, 12678 (1999).

${ }^{20}$ C. Journet, W. K. Maser, P. Bernier, A. Loiseau, M. Lamy de la Chapelle, and S. Lefrant, Nature (London) 388, 756 (1997).

${ }^{21}$ E. Muñoz, W. K. Maser, A. M. Benito, M. T. Martínez, G. F. de la Fuente, A. Righi, J. L. Sauvajol, E. Anglaret, and Y. Maniette, Appl. Phys. A: Mater. Sci. Process. 70, 145 (2000).

${ }^{22}$ A. Jorio, A. G. Souza Filho, G. Dresselhaus, M. S. Dresselhaus, A. K. Swan, M. S. Ünlü, B. B. Goldberg, M. A. Pimenta, J. H. Hafner, C. M. Lieber, and R. Saito, Phys. Rev. B 65, 155412 (2002).

${ }^{23}$ A. Kasuya, Y. Sasaki, Y. Saito, K. Tohji, and Y. Nishina, Phys. Rev. Lett. 78, 4434 (1997).

${ }^{24}$ P. Corio, M. L. A. Temperini, P. S. Santos, J. V. Romero, J. G. Huber, C. A. Luengo, S. D. M. Brown, M. S. Dresselhaus, G. Dresselhaus, M. S. S. Dantas, C. F. Leite, F. Matinaga, J. C. Gonzalez, and M. A. Pimenta, Chem. Phys. Lett. 350, 377 (2001).

${ }^{25}$ L. Alvarez, A. Righi, S. Rols, E. Anglaret, and J. L. Sauvajol, Chem. Phys. Lett. 320, 441 (2000).

${ }^{26}$ M. T. Martínez, M. A. Callejas, A. M. Benito, M. Cochet, T. Seeger, A. Anson, J. Schreiber, C. Gordon, C. Marhic, O. Chauvet, J. L. G. Fierro, and W. K. Maser, Carbon 41, 2247 (2003).

${ }^{27}$ PingHeng Tan, Long An, LuQi Liu, ZhiXin Guo, R. Czerw, D. L. Carroll, P. M. Ajayan, Nai Zhang, and HongLi Guo, Phys. Rev. B 66, 245410 (2002).

${ }^{28}$ K. Nakamura and M. Kitajima, Phys. Rev. B 45, 78 (1992).

${ }^{29}$ R. J. Nemanich and S. A. Solin, Phys. Rev. B 20, 392 (1979).

${ }^{30}$ T. Pichler, M. Matus, J. Kürti, and H. Kuzmany, Phys. Rev. B 45, 13841 (1992).

${ }^{31}$ K.-A. Wang, Y. Wang, P. Zhou, J. M. Holden, S. L. Ren, G. T. Hager, H. F. Ni, P. C. Eklund, G. Dresselhaus, and M. S. Dresselhaus, Phys. Rev. B 45, 1955 (1992).

${ }^{32}$ R. Saito, T. Takeya, T. Kimura, G. Dresselhaus, and M. S. Dresselhaus, Phys. Rev. B 57, 4145 (1998).

${ }^{33}$ A. G. Souza Filho, A. Jorio, Ge. G. Samsonidze, G. Dresselhaus, M. A. Pimenta, M. S. Dresselhaus, A. K. Swan, M. S. Ünlü, B. B. Goldberg, and R. Saito, Phys. Rev. B 67, 035427 (2003).

${ }^{34}$ A. Jorio, M. A. Pimenta, A. G. Souza Filho, Ge. G. Samsonidze, A. K. Swan, M. S. Ünlü, B. B. Goldberg, R. Saito, G. Dresselhaus, and M. S. Dresselhaus, Phys. Rev. Lett. 90, 107403 (2003). If we impose the exponent $x=1.4$ to fit data of Fig. 2(a) we obtain $\nu_{0}=1514.8 \mathrm{~cm}^{-1}$ and $C=-0.073072 \mathrm{~cm}^{0.4}$.

${ }^{35}$ R. Saito, G. Dresselhaus, and M. S. Dresselhaus, Phys. Rev. B 61, 2981 (2000). 